\title{
Ordenanzas en base metabólica; el replanteo de las ordenanzas de densificación en tres urbanizaciones del Vallés (Barcelona).
}

\author{
Roger-Joan Sauquet Llonch ${ }^{1}$, Isabel Castiñeira Palou ${ }^{2}$, \\ Nieves Gómez Priego ${ }^{3}$, Chantal Dones Muñoz ${ }^{4}$ \\ ${ }^{1}$ Departamento de Proyectos Arquitectónicos. \\ ${ }^{2}$ Departamento de Urbanismo y Ordenación del Territorio. ${ }^{34}$ Postgraduadas. \\ Universitat Politècnica de Catalunya, Barcelona, España \\ E-mail: ${ }^{1}$ Roger-joan.sauquet@upc.edu, ${ }^{2}$ Isabel.castineira@upc.edu, \\ ${ }^{3}$ nievesgpri@gmail.com, ${ }^{4}$ chantaldones@gmail.com
}

\begin{abstract}
Resumen. Las áreas de baja densidad residencial en Cataluña toman el nombre de 'urbanizaciones'. Algunas nacidas a principio de siglo XX y muchas otras de forma ilegal durante el tardo-franquismo, estos asentamientos se caracterizan por las casas unifamiliares en medio de parcelas de entre 400 y 1.000 metros cuadrados. A pesar de todas las patologías urbanas y ambientales atribuidas a este tipo de tejidos hay indicadores como el de la biodiversidad o el de la calidad ambiental del aire que son notablemente mejores que los de la ciudad compacta. Así mismo, es de los pocos espacios urbanos dónde aún se puede experimentar, desde de la rehabilitación, nuevos tipos residenciales de densidad media que rompan la dicotomía casa - bloque.

Estas áreas crecen a partir de las necesidades vitales de los habitantes que construyen cuando necesitan más espacio. Aprovechando esta realidad metabólica, el artículo se plantea hasta qué punto la forma de planificar el urbanismo 'clásico', que interpone límites meramente volumétricos a la obra nueva o a las ampliaciones, es efectiva, o bien podría ser substituida por la regulación compleja de un intercambio de intereses entre el habitante, que necesita obtener más superficie y más rendimiento de su parcela, y la globalidad, que necesita mejorar la sostenibilidad ambiental y social de este tipo de tejidos. Desde distintas plataformas docentes de la ETSAV, Universidad Politécnica de Cataluña (UPC) se ha estudiado pormenorizadamente tres áreas de baja densidad de la comarca barcelonesa del Vallès, proponiendo un sistema normativo con el que, mediante condicionantes, se regulan las aspiraciones de crecimiento de los habitantes limitándoles o premiándoles en función de las bondades sociales y ambientales de la intervención arquitectónica que pretenden realizar.
\end{abstract}

Palabras clave: urbanizaciones, normativa, ordenanzas, crecimiento metabólico, baja densidad.

Introducción a la baja densidad en Cataluña, específicamente en la región metropolitana de Barcelona

El territorio catalán, especialmente las áreas costeras y las áreas de montaña próximas a las capitales, han sufrido el fenómeno llamado "urbanizaciones" cuya principal característica es, precisamente, la falta de urbanización y sobre todo la falta de urbanidad. Tiene su origen en parcelaciones sobre suelo rústico, situadas en parajes de mayor o menor interés paisajístico, a menudo en áreas de grandes pendientes y acceso complicado. Aunque las hay anteriores y posteriores, el punto álgido de estas, mal llamadas, "urbanizaciones" se 
concentra en los años 60 , constituyendo una oferta de "segunda residencia" o residencia vacacional para familias con domicilio en las principales ciudades. La oferta inicial se formaliza con la venta de parcelas y la posterior construcción de viviendas para vacaciones, ligadas a la playa o a los pueblos de montaña, pero que, en general, se disponen alejadas de la costa y del pueblo, constituyendo unidades residenciales aisladas en el territorio, con falta de servicios y con dificultad de acceso

A partir de los años 80, los Ayuntamientos democráticos realizaron un enorme esfuerzo para regularizar la situación de estos ámbitos, tanto desde el punto de vista funcional, mejorando accesos e implementando los servicios y urbanización que muchas de ellas carecían, como des del punto de vista urbanístico y legal, a través de un reconocimiento en el planeamiento municipal y la implementación, en la medida que era posible, de espacios libres y reservas para equipamientos. Ya en los últimos años del siglo $\mathrm{XX}$ estas residencias vacacionales se transformaron, en muchos casos, en residencia permanente de los propios propietarios, ya jubilados, o de algún familiar. Todo ello incrementó la carga municipal, especialmente en lo que se refiere a mantenimiento e implementación de servicios así como el acceso a los servicios básicos.

En las áreas centrales, en los ámbitos más densamente pobladas de la Región Metropolitana de Barcelona, el fenómeno se muestra con mayor contundencia, conviviendo áreas similares a las anteriormente descritas con otras que se disponen como áreas de crecimiento de las ciudades o integradas a ellas, constituyendo áreas residenciales de baja densidad, ocupando importantes extensiones próximas a la red de comunicaciones, especialmente autopistas, disponiendo de una relación de proximidad con la ciudad de Barcelona u otras ciudades importantes. En este caso han supuesto una opción residencial para familias que han optado por ofertas de una mayor calidad de vida familiar, ligada a la naturaleza con ventajas de tamaño de la propia vivienda y acceso a jardín, opción prácticamente inexistente en Barcelona. Todo esto a cambio de una dependencia funcional en relación al trabajo y a los equipamientos y servicios, la mayor parte de los residentes en estas áreas se desplazan diariamente en coche entre residencia y trabajo o estudio, en tiempos muy superiores a la duración teórica del trayecto.

También existe una tercera familia, menos abundante, de barrios de baja densidad que nacen como actuaciones con ubicación y finalidades específicas, dirigidas a determinadas comunidades e integrando un importante contenido social. En estos casos son proyectos unitarios inspirados en el modelo de la ciudad jardín, reconocidos por la tesis doctoral de Jordi Franquesa como "comunidades jardín"1. El objetivo de dar vivienda de calidad a una determinada comunidad, normalmente vinculada a la producción industrial de la primera mitad del siglo pasado, se muestra en estos barrios residenciales con la presencia de equipamientos, lugares de referencia comunitaria como plazas y espacios libres, un elevado nivel de urbanización, regularidad en el trazado parcelario y una definición precisa de las tipologías residenciales. Generalmente se trata de barrios de construcción unitaria y con presencia de diversas tipologías, en la mayoría de los casos unifamiliares con jardín. En la actualidad se ha reconocido un valor urbanístico y ambiental a estas comunidades, constituyendo ámbitos que gozan de algún tipo de valoración patrimonial en los correspondientes municipios, cuyas ordenanzas tienden a conservar los espacios, usos y tipologías en sus condiciones originales.

\section{La intensificación de las "urbanizaciones" de la Región Metropolitana de Barcelona}

E1 Plan Territorial Metropolitano de Barcelona clasifica estas áreas en función de su ubicación, ocupación y proximidad a los núcleos urbanos o redes de transporte para determinar si se requiere su intensificación o su desaparición ${ }^{2}$. Desde el Máster Universitario en Arquitectura de la ETSAV, de la Universidad Politécnica de Cataluña (UPC), así como desde algunos talleres del Grado en Arquitectura, se ha planteado el reto de la intensificación como herramienta para la mejora del hábitat urbano. El hecho de que nuestra propia escuela se ubique en Sant Cugat del Vallès, ciudad muy cercana a 
Fig. 1. Comparación entre el funcionamiento conceptual de

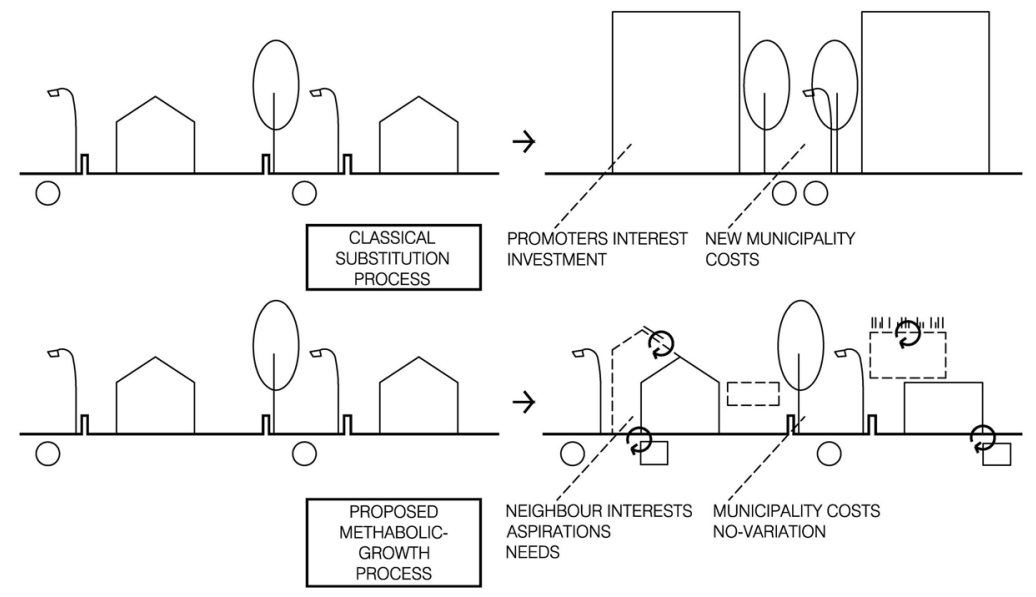
versus uno de transformación (fuente autor).

Barcelona con un marcado predominio de áreas de baja densidad, nos confiere una especial sensibilidad hacia el tema. En concreto se han tratado casos del segundo y tercer modelo de "urbanizaciones" (según lo descrito en el punto anterior), donde los tejidos residenciales tienen cierto grado de integración social y funcional a los núcleos urbanos.

Los problemas que presentan actualmente estos conjuntos residenciales se pueden considerar desde varios puntos de vista: La masiva ocupación del territorio que suponen las urbanizaciones de baja densidad, los problemas de accesos y accesibilidad a servicios y equipamientos, la dificultad de implementar transporte público en estos ámbitos, entre otros problemas que son abordados desde estudios teóricos y desde los planes municipales. Cabe destacar los dos foros de debates organizados y posteriormente publicados por la Diputación de Barcelona mediante los libros editados por Francesco Indovina y Francesc Muñoz respectivamente ${ }^{3}$.

Hay que decir, también, que la intensificación de la baja densidad es una tarea compartida por muchos países del arco mediterráneo norte, así como en espacios de mayor tradición de hábitat disperso como son los países anglosajones, especialmente Estados Unidos. Sin embargo, algunas experiencias mostradas en algunos manuales de urbanismo explicitan un cierto fracaso al introducir una estrategia de substitución urbana y no de crecimiento complejo ${ }^{4}$.

En este sentido, la estrategia estudiada desde la ETSAV, parte del principio de transformación, o "reparación dinámica" de Sennett, y atenderá específicamente a las posibilidades de transformación desde el tejido social y la estructura de la propiedad existentes en el lugar (Fig. 1), procurando convertir la densificación en una oportunidad para la implementación de equipamientos de proximidad, así como la posible recuperación, o creación, de áreas urbanas de referencia o de centralidad local. Se trata de convertir estas "urbanizaciones" en barrios, entendiendo el barrio como un elemento urbano con vida propia, servicios propios, pero integrada física y socialmente a una población.

La medida busca, obviamente, aumentar la masa crítica de estas áreas, con la intención de convertir en eficiente el transporte público y en rentable el comercio. Pero también, no caer en la fácil dualidad "casa" o "bloque" como arquetipos habitacionales mediterráneos, sino explorar en la transformación de las viviendas a cuatro vientos modelos de hábitat complejos que incorporen principios urbanos cercanos al urbanismo ecológico o ecosistémico aprovechando los valores positivos de la baja densidad en cuanto a biodiversidad y calidad del aire ${ }^{6}$.

\section{Hipótesis y justificación metodológica - método de trabajo -, resolución y condicionantes}

La estrategia planteada en los casos estudiados por la ETSAV, parten, como explicitábamos, de los elementos más pequeños del engranaje urbano: las parcelas y las viviendas. A partir 
de una exploración de las posibilidades de transformación de estos elementos, así como de sus actores - propietarios o residentes -, se introducen factores y condicionantes de transformación que tengan la capacidad de restaurar los déficits ambientales de nuestras ciudades - hay que entender que la construcción y e consumo energético de las viviendas son una importante contribución al consumo energético global $^{7}-$, así como los déficits de urbanidad: el excesivo encapsulamiento del hábitat, la inexistencia de espacios de relación, la especialización de la calle en atributos viarios.

Por otra parte, el título del artículo hace referencia a un instrumento urbanístico clásico, las ordenanzas, que ha contribuido al desarrollo equilibrado de los tejidos urbanos en todas nuestras ciudades, cuyos parámetros, con definiciones numéricas muy diversas, se han consolidado como factores íntimamente ligados a la forma e imagen urbana. El estudio llevado a cabo invita a una reflexión sobre la naturaleza de este instrumento, criticando el hecho que responda básicamente a la implantación y volumetría de las edificaciones, siento insensible al grado de sostenibilidad de la intervención arquitectónica que cada propietario o habitante promueve, tratando por igual intervenciones con gastos de $\mathrm{CO} 2$ distintos, con grados de impermeabilización de la parcela distintos, con contribuciones a la biodiversidad distintas, o con relaciones entre la edificación, la calle y los vecinos distintas, para decir sólo algunos parámetros.

La misión de las ordenanzas aplicadas mayoritariamente en las ciudades catalanas tiene que ver con un modelo de planeamiento "clásico", que dibuja la ciudad, con clara influencia "rossiana", o de la "Escuela de Barcelona" en su aplicación regional ${ }^{9}$. Por el contrario, las ordenanzas exploradas desde la ETSAV confieren un giro en la idea de intervención urbanística incorporando el concepto de "sistema abierto" que Sennett teoriza para el espacio público ${ }^{10}$, así como el concepto de crecimiento metabólico introducido a principios y mediados del siglo XX por autores como Geddes o Mumfort y planes como Bradacre City de Frank Lloyd Wright $^{11}$.
Las ordenanzas aquí planteadas se conciben como una plataforma para mediar un intercambio de intereses, entre lo que pide el promotor y lo que demanda la colectividad. Los intereses de los promotores, que en el caso de las "urbanizaciones" suelen ser los mismos habitantes, son, básicamente:

- $\quad$ el crecimiento de su espacio habitable sobrepasando gálibos preestablecidos mediante normativas - motivados por los cambios en la composición familiar, en las formas de habitar

- las posibilidades dividir, de alquilar o vender parte de su vivienda - necesidad de obtener recursos, imposibilidad de los hijos de emanciparse

Por otro lado, los intereses del colectivo, inmediato o global, representado por la administración local o por las entidades no gubernamentales, son, entre otros:

- la densificación del parque edificado

- la reducción de la huella ecológica

- $\quad$ la mejora de la biodiversidad del área urbana

- la mejora de la habitabilidad

- la mejora del espacio público

- $\quad$ el aprovechamiento de los recursos

- la disminución de los residuos.

La idea de este cambio en el concepto de ordenanza queda reflejado en el gráfico de la figura 2.

En este sentido, las condiciones de trasformación de las parcelas-viviendas que las
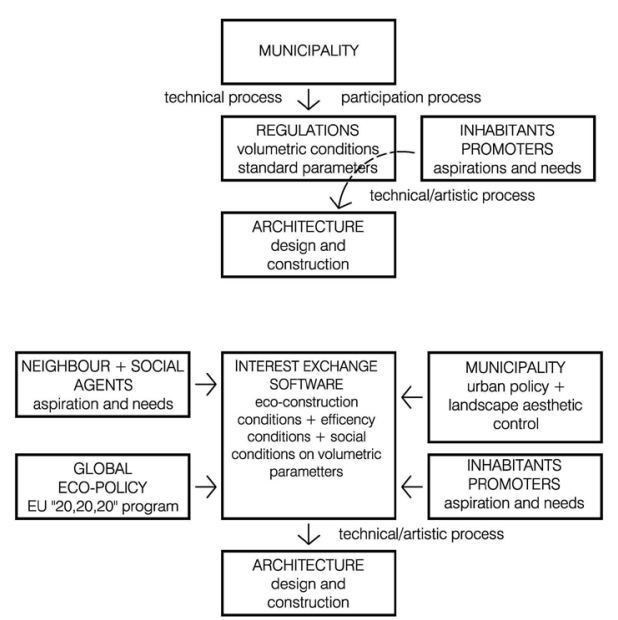

Fig. 2. Comparación entre el funcionamiento de una ordenanza clásica y una ordenanza concebida como un sistema de intercambio de intereses (fuente autor). 
nuevas ordenanzas deben contemplar tienen que ofrecer:

la posibilidad de mejorar las condiciones ambientales de la propia parcela, del espacio edificado y del no edificado, considerando el objetivo de que las actuaciones que en ella se realicen tengan una repercusión positiva en la propia edificación y en la comunidad

- la posibilidad de dar respuesta a composiciones familiares diversas

la posibilidad de densificación manteniendo las calidades ambientales

la posibilidad de incorporar usos no residenciales al servicio de la diversidad y la comunidad

Los condicionantes a las intervenciones en la parcela-vivienda, reguladas bajo esta nueva ordenanza, tendrían que ir acompañados de otros instrumentos de planeamiento que resuelvan medidas de carácter estructural, como puede ser la mejora de las circulaciones, de los espacios libres, la definición de áreas con distinto grado de densificación, si es necesario, la disposición de puntos de mini-centralidad donde convivan tipologías plurifamiliares con espacios urbanos relacionados con el transporte público, comercios y equipamientos.

Resumiendo, la incorporación de criterios metabólicos a las ordenanzas, establecidos con el rigor suficiente, pero también con suficiente flexibilidad, además de su principal objetivo de mejorar las condiciones metabólicas de la ciudad incidiendo directamente en el aprovechamiento de los recursos: ciclo del agua, energías renovables de forma pasiva o activa, gestión de los residuos, reducción de tránsito, entre otros, puede contribuir a la implementación de otras ventajas que afecten a las condiciones de diversidad funcional y social de las comunidades. En definitiva, la investigación sobre otras formas de definición de ordenanzas se basa en la coordinación de las ventajas individuales: adaptación funcional, diversificación, mayor confort y ahorro energético con otras ventajas que afectan a la comunidad, centradas particularmente en la diversificación, la mejora ambiental y un mejor aprovechamiento de los recursos. El estudio de nuevas ordenanzas tiene como objetivo el establecimiento de correspondencias entre las mejoras ambientales que puede ofrecer la mejora o rehabilitación, con las posibilidades de renovación y edificación.

\section{Tres casos de estudio en la comarca del Vallès, Región Metropolitana de Barcelona}

Como se mencionaba anteriormente, la investigación aquí presentada se ha desarrollado en distintas plataformas docentes de la ETSAV. Por un lado, la intensificación de los tejidos de baja densidad fue tratado en un taller de último año del Grado en Arquitectura del curso 2014/2015. Este taller propuso medidas estratégicas de densificación, a nivel de estructura urbana, del conglomerado formado por las urbanizaciones de Valldoreix, Mira-Sol y La Floresta en Sant Cugat del Vallés. Posteriormente, un área perteneciente a una de estas urbanizaciones, Mas-Gener, fue estudiada pormenorizadamente por el taller del Máster Universitario en Arquitectura del curso $2015 / 2016$. Se investigaron cuestiones relativas al grado de consolidación de los valores de edificabilidad y ocupación de las parcelas y se plantearon medidas de mejora del espacio urbano y la introducción de equipamientos. Finalmente, el último espacio abordado, la urbanización/colonia industrial de Nostra Llar en Sabadell, formó parte también del taller del Máster Universitario en Arquitectura durante el curso 2017/2018. En este último eslabón, se concretaron parámetros y condicionantes de crecimiento organizados a modo de ordenanza. Dedicaremos gran parte de la segunda parte del artículo a este último caso.

Vallodreix, Mira-Sol y La Floresta en Sant Cugat del Vallès

Este conjunto de urbanizaciones, situados a la falda norte de la sierra de Collserola, tiene una población de 23.000 habitantes y una superficie de $21 \mathrm{~km} 2$. Las tres urbanizaciones tenían hasta hace veinte años una razón de ser vacacional que ha pasado a primera residencia en casi su totalidad. A nivel físico, Valldoreix y Mira-sol se encuentran en un terreno bastante llano, dominado por torrentes que cruzan el área urbana, mientras que La Floresta está situada en la ladera de Collserola con parcelas 


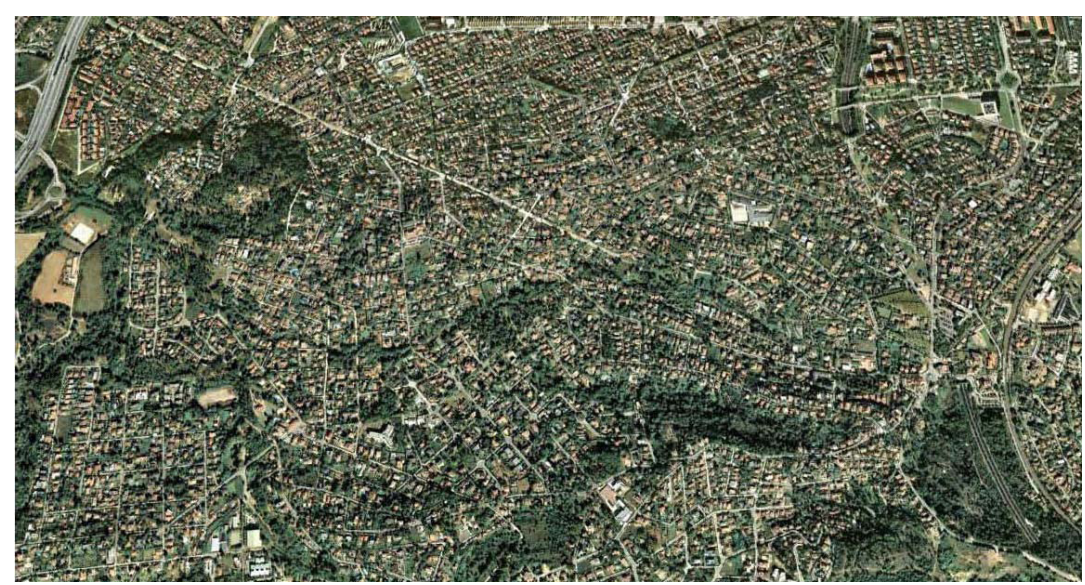

Fig. 3. Imagen aérea del complejo urbano formado por las urbanizaciones de Valldoreix y Mira-sol en Sant Cugat del Vallès (fuente: www.google.com/maps).

en terrenos de fuerte pendiente. El viario de las tres mantiene cierta conexión, especialmente entre Valldoreix y Mira-sol (figura 3).

El taller estaba enfocado a encontrar estrategias de distribución de la densidad en función de la realidad urbana de cada uno de estos "barrios" teniendo en cuenta cuestiones como el funcionamiento viario, la ubicación de las paradas de autobús, el emplazamiento del comercio, la existencia de pequeñas áreas de centralidad. La conclusión del curso está reflejada en la figura 4 que define 5 estrategias de densificación:

-Estrategia 1 - Densificación de alta intensidad concentrada en un centro. Indicada para áreas urbanas donde la existencia de una cierta centralidad de combina con parcelas de oportunidad, abandonadas o públicas, en las que, a parte de generar vivienda, se genere actividad comercial y de servicios.

-Estrategia 2 - Densificación progresiva a partir de un centro. Sigue la lógica de los desplazamientos desde las paradas de autobús o tren generando un degradado de la densidad desde las áreas más alejadas a este foco de intensidad hasta las parcelas contiguas a él.

-Estrategia 3 - Densificación concentrada en una calle. Algunas calles de Valldoreix actúan de "desguace" del viario y presentan una tímida actividad comercial. Esta estrategia permitiría la densificación de las parcelas que dan a la calle así como la posibilidad de construir fachadas con espacios comerciales.

-Estrategia 4 - Densificación asimétrica en calles con muros de contención. La urbanización La Floresta presenta parcelas muy desniveladas con muros de contención precarios que amenazan el espacio público. Lo planteado allí fue permitir la sustitución de este muro deteriorado por un muro con grosor suficiente para albergar vivienda i/o comercio.

-Estrategia 5 - Híper-densificación en el espacio público. Es quizá la estrategia que

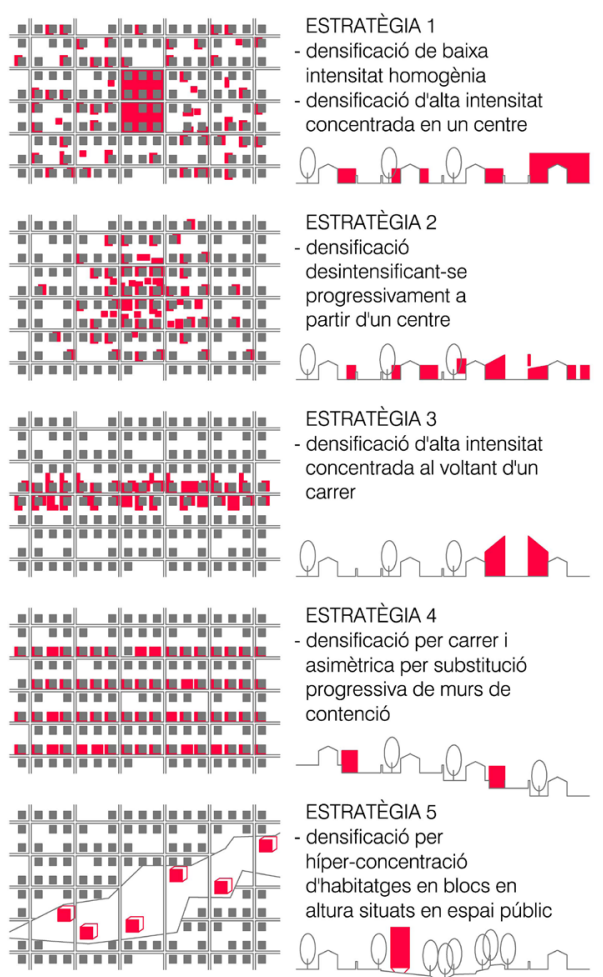

Fig. 4. Estrategias de implantación de la densidad según morfología y funcionamiento urbano de la "urbanización" desarrollados en el Taller $\mathbf{E} 1$ del curso 2012/2013 de la ETSAV (fuente autor). 


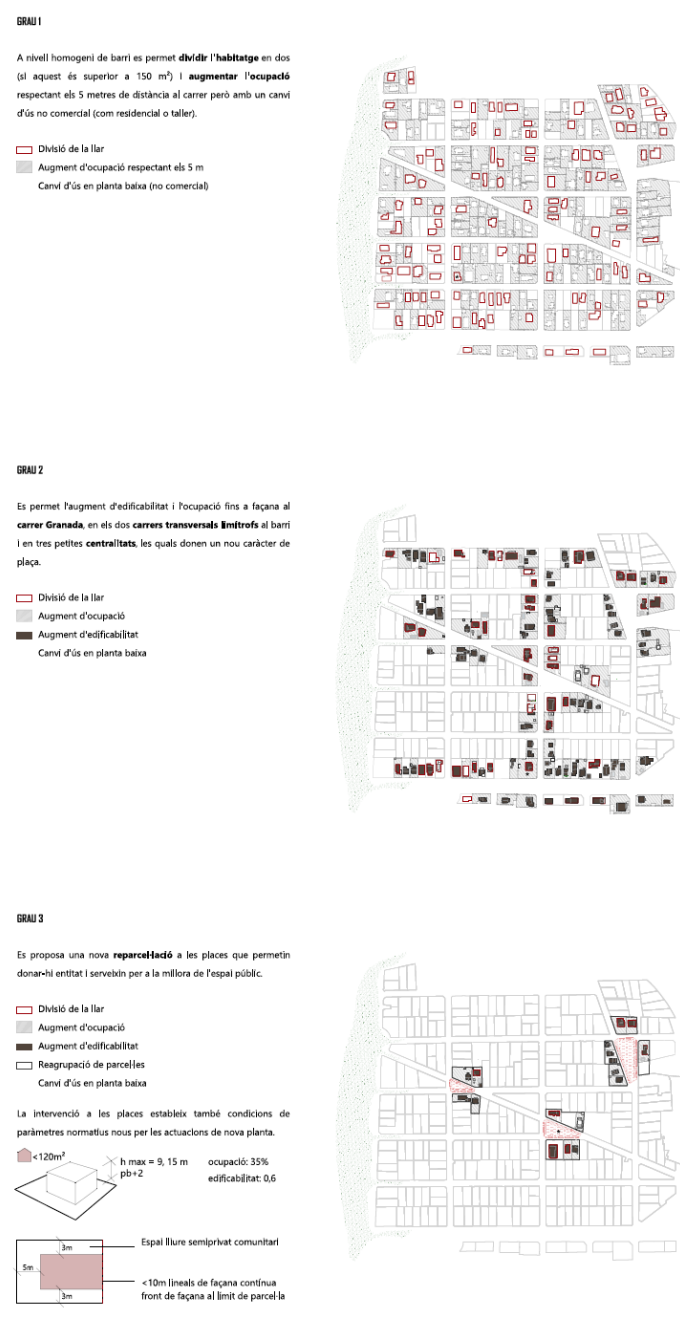

menos sigue la filosofía de la investigación, pero parte de la reflexión sobre las áreas de espacio verde, especialmente los parques urbanos, situados en este tipo de urbanizaciones que tienen el entorno natural cercano (el Parque Natural de Collserola) y que muchas de las viviendas están rodeadas de jardín. El deterioro de los parques, más su titularidad pública, permiten plantear cambios normativos que posibilitan la introducción de pequeños bloques con viviendas para colectivos vulnerables, especialmente los jóvenes.

\section{Urbanización Mas-Gener en Sant Cugat del Vallès}

Mas-Gener es una urbanización de unas 450 familias que forma parte de Mira-Sol. Su autonomía geográfica y urbana lo convierten en un adecuado caso práctico. El trabajo en el taller consistió en detectar minuciosamente las posibilidades de crecimiento partiendo del análisis de la edificabilidad y la ocupación de cada parcela y de las oportunidades que ofrecen su funcionamiento viario $\mathrm{y}$ su estructura de equipamientos. Igualmente, la contigüidad con un área de crecimiento aún para desarrollar permitía equilibrar carencias como el envejecimiento de la población, construyendo nuevas viviendas, o la falta de algunos servicios. En conclusión, la situación del parque edificado real en comparación con el planeamiento era de una situación de ocupación prácticamente agotada pero una baja consolidación de la edificabilidad, por lo tanto se plantearon cuatro actuaciones (figura 5):

-Actuación 1 - bajo la condición de dividir la vivienda en dos o tres, permitir ocupaciones superiores a las establecidas en el planeamiento pero sin agotar la edificabilidad fijada por el

Fig. 5. (izquierda, arriba) Conjunto de actuaciones para densificar el barrio de Mas-Gener del proyecto PFC Rehabitar la baixa densitat : noves condicions de ciutat als barris de Mas Gener i Can Cabassa, Sant Cugat del Vallès de la estudiante Clara Grenzner (fuente: https://upcommons.upc.edu/ handle/2117/107908).

Fig. 6. (izquierda, abajo) Evolución de la densidad según las propuestas de regeneración urbana del barrio de Mas-Gener según el proyecto PFC Rehabitar la baixa densitat (ídem anterior). 
mismo.

-Actuación 2 - en las calles que configuran el sistema viario principal, permitir el aumento de la edificabilidad a cambio de subdividir las viviendas y generar fachada a la calle con fines comerciales.

-Actuación 3 - en determinados puntos de centralidad permitir la junción parcelaria a cambio de introducir nuevas viviendas y generar espacios para la actividad comercial.

-Actuación 4 - utilizando el área urbanizable para desarrollar contigua, recuperar valores cercanos a la ciudad compacta en cuanto a densidad, proximidad a equipamientos, actividad comercial.

La evolución de la densidad tras aplicar las cuatro estrategias aparece reflejada en el gráfico (Fig. 6).

\section{Barrio de Nostra Llar en Sabadell}

El tercer proyecto/caso de estudio se desarrolla en Nostra Llar de Sant Oleguer, un barrio residencial de baja densidad de Sabadell. Ubicado, en el momento de su construcción, en las afueras de la ciudad, actualmente constituye un barrio singular, próximo al centro y vías principales y con una relación directa con el Río Ripoll, actualmente recuperado como eje verde territorial que contiene equipamientos y zonas verdes (Fig. 7).

Fue construido en los años 60 promovido por los dueños de una fábrica de la industria textil local para alojar a sus trabajadores. Se concibió como un barrio de "casas baratas" unifamiliares inspirado en la ciudad jardín británica. Las viviendas, muy similares entre sí, son principalmente de dos plantas y se agrupan en bloques de dos o cuatro unidades. Rondaban los $60 \mathrm{~m} 2$ y estaban pensadas para familias de 4 o 5 miembros. Actualmente, gran parte de las viviendas están habitadas por la población original, envejecida y con una ocupación media de 2 a 4 personas por vivienda. Las casas están poco aprovechadas $\mathrm{y}$, a menudo, las condiciones físicas resultan inadecuadas para las necesidades la población.

En su historia, las viviendas se han transformado dando respuesta a diversas necesidades en la evolución de la familia original, especialmente en lo que se refiere

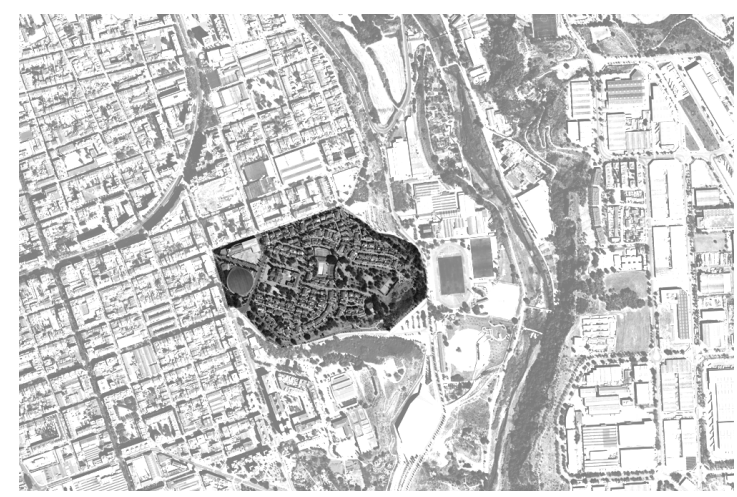

Fig. 7. Imagen aérea manipulada para señalar el Barrio de Nostra Llar en Sabadell (fuente: www. google.com/maps).

a la habilitación de nuevos espacios mediante ampliaciones que se han producido desordenadamente, sin proyecto ni coherencia entre ellas. Dicha necesidad se recogió en la normativa municipal, que las admite limitándolas a $35 \mathrm{~m} 2$ dispuestas en planta baja de la parte posterior de la parcela. Sin embargo, la realidad no se adapta a dicha normativa, y, aunque se cumpliera, la ocupación del espacio posterior de la parcela en su totalidad, empeora la ventilación e iluminación de la vivienda. A su vez se generan barreras visuales cuando estas ampliaciones limitan con la calle. Al analizar el estado de las viviendas del barrio, se constató que la normativa se incumplía en muchos casos, superando el límite de superficie y construyendo en el jardín frontal de la vivienda $\mathrm{y}$ en la planta primera.

Este análisis se ha contrastado con la comunidad de vecinos mediante procedimientos participativos y entrevistas a sus miembros. De esta manera, se identifican a su vez, exigencias de la normativa actual que no son respetadas pero resultan importantes para el bienestar particular y de la comunidad y se crea un diálogo sobre nuevas posibilidades de intervención que respecten las necesidades de los vecinos y permitan generar comunidades más sanas y sostenibles desde el punto de vista arquitectónico, social y medioambiental.

La ordenanza vigente, fue redactada con la misión de "ordenar" el barrio y tiene una clara vocación estética, en el sentido que parte de una imagen congelada de lo que debería ser el barrio definiendo, además de los volúmenes 


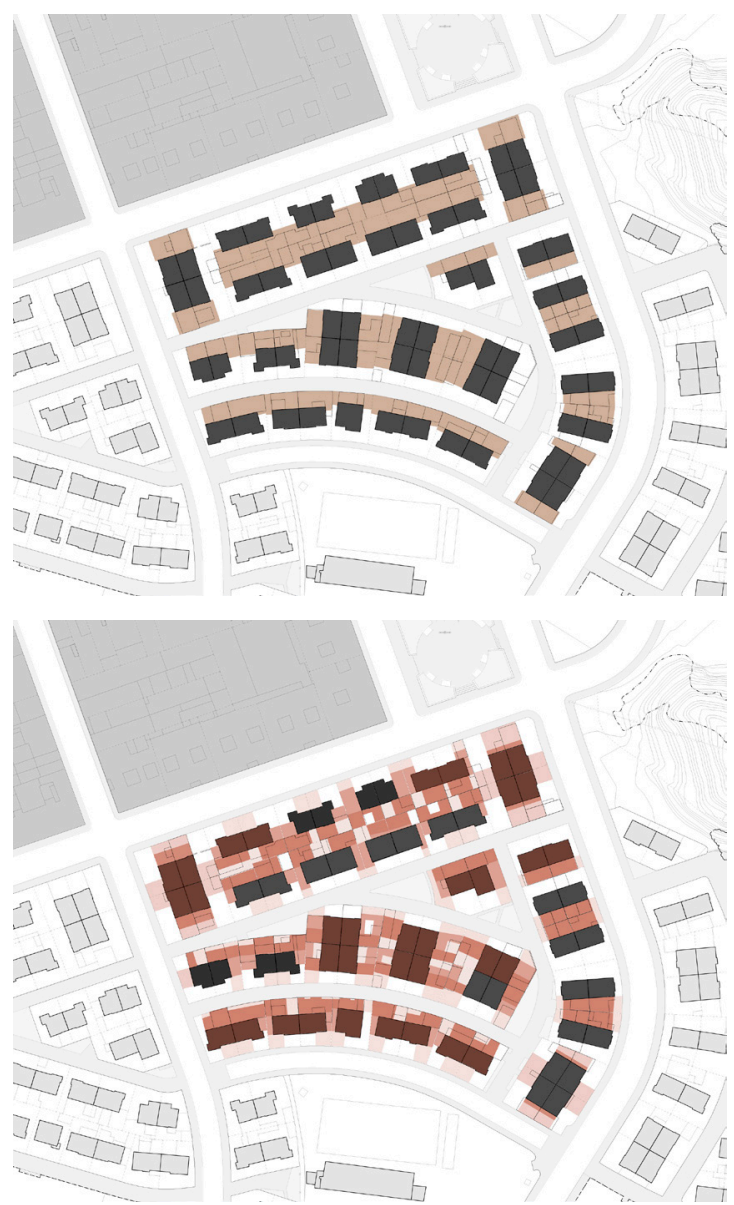

Fig. 8. (arriba) Gálibos según la normativa vigente en el conjunto Nostra Llar de Sabadell (fuente autoras). Fig. 9. (abajo) Gálibos según la normativa propuesta en el conjunto Nostra Llar de Sabadell (fuente autoras).

permitidos, sus colores, la composición de las ventanas, los sistemas constructivos de fachadas y cubiertas. Desde el taller se pretendió dar un giro a esta normativa dando más importancia a la conservación y puesta al día de los valores sociales de la comunidad original que al aspecto arquitectónico ya transformado.

En este sentido, nueva normativa pretende ser un intercambio de intereses particulares y sociales. Aquellos vecinos que más colaboren con los intereses colectivos del barrio, serán aquellos que contarán con mayores beneficios a nivel privado. De esta forma, se sustituye el sistema actual de norma-incumplimientopenalización por guía-cumplimentorecompensa.

Tras un análisis de las viviendas y sus diferentes orientaciones, relaciones con el espacio público y edificaciones existentes, se crean unos nuevos gálibos. Dentro de estos, existen diversas opciones de ocupación limitadas por el estudio de obstrucción solar a otras viviendas. Y, respetando estos criterios, se plantean gálibos de más ocupación y edificabilidad y gálibos de menos (Fig. 8 y 9).

Con la finalidad de hacer comprensible la nueva ordenanza, se establecen nuevos criterios de carácter ambiental o de uso beneficiosos para la comunidad, cuyo cumplimiento por parte de los particulares conlleva una ganancia "puntos" que se traducen en un aumento del gálibo o volumen regulador y en un incremento de la superficie ampliable permitida $(35 \mathrm{~m} 2)$. El sistema de "puntos" requiere de un análisis pormenorizado de la intervención que cada vecino quiera realizar en su vivienda. En función de la puntuación las posibilidades de ampliación de las viviendas será mayor o menor (Fig. 10).

Para gestionar el incremento de superficie de las viviendas, es necesario encontrar un módulo o medida que dé valor a los puntos. Teniendo en cuenta las dimensiones de las estancias de una vivienda, se decide que un punto corresponderá a $3 \mathrm{~m} 2$. (ver la comparación entre el esquema conceptual de la normativa actual, Fig. 11, y el de la normativa propuesta, Fig. 12)

La puntuación se obtiene en base a ocho ámbitos de actuación, que corresponden a las

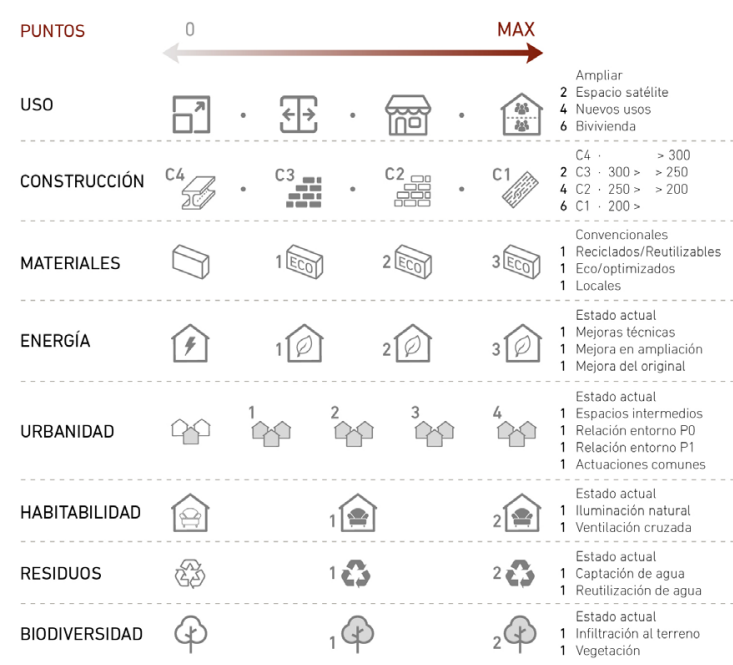

Fig. 10. Sistema de puntuación según intervención arquitectónica a las parcelas y viviendas existentes, según la normativa propuesta en el conjunto Nostra Llar de Sabadell (fuente autoras). 
necesidades sociales identificadas en el análisis

anterior, y que quedan reflejados en esta tabla:

\begin{tabular}{|c|c|}
\hline AMBITO & OBJETIVO \\
\hline 1. Uso & $\begin{array}{l}\text { Re-densificar, responder a una comunidad cambiante, } \\
\text { heterogénea y con necesidades diversas. }\end{array}$ \\
\hline 2. Construcción & $\begin{array}{l}\text { Crecimiento sostenible contabilizado en base a las emisiones de } \\
\mathrm{CO}_{2} \text {. }\end{array}$ \\
\hline 3. Materiales & $\begin{array}{l}\text { Abarcar aspectos no computables en las emisiones de } \mathrm{CO}_{2} \text { que } \\
\text { contribuyen a la sostenibilidad, durabilidad y aumento del ciclo } \\
\text { de vida de estos. }\end{array}$ \\
\hline 4. Energía & $\begin{array}{l}\text { Reducir el consumo de energía no renovable e idealmente } \\
\text { generar una menor demanda mediante estrategias pasivas. }\end{array}$ \\
\hline 5. Urbanidad & $\begin{array}{l}\text { Fomentar la seguridad urbana, crear comunidad, revitalizar la } \\
\text { calle. }\end{array}$ \\
\hline 6. Habitabilidad & $\begin{array}{l}\text { Mejorar las condiciones de funcionamiento de las viviendas y } \\
\text { con ello la calidad de vida de los habitantes. }\end{array}$ \\
\hline 7. Residuos & $\begin{array}{l}\text { Reducir la huella medioambiental y conseguir un consumo más } \\
\text { responsable de los recursos. }\end{array}$ \\
\hline 8. Biodiversidad & $\begin{array}{l}\text { Reintroducir el concepto de ciudad jardín de forma consciente } \\
\text { con su impacto en el medio. }\end{array}$ \\
\hline
\end{tabular}

Existen diversos grados de importancia y facilidad de cumplimiento de estos ámbitos de actuación que ponderan su valor. Estos se evalúan en algunos casos asociando un número de puntos a los diferentes niveles de actuación, como es en el caso de la introducción de nuevos usos, como "habitaciones satélite"12, comercios o áreas de trabajo y bi-viviendas,

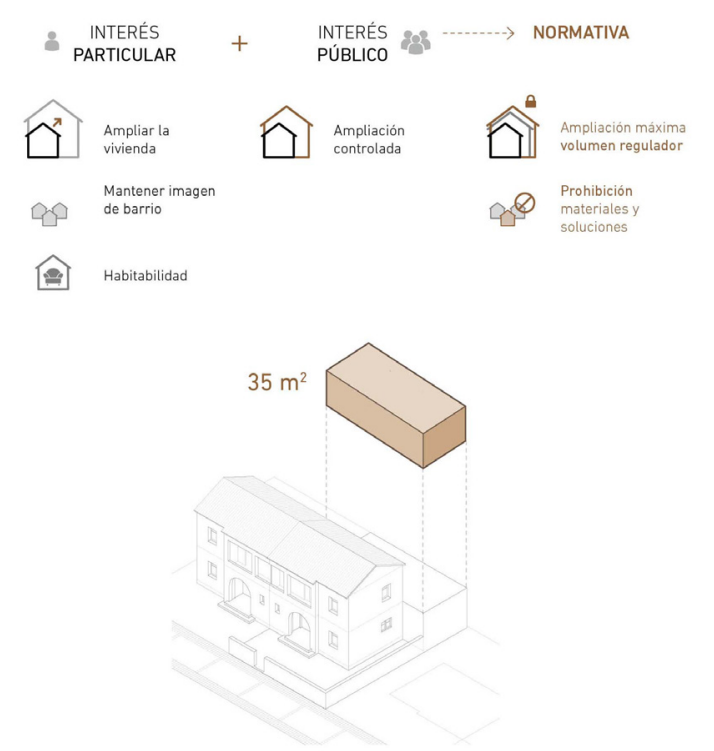

Fig. 11. Esquema conceptual de funcionamiento de la normativa vigente en el barrio de Nostra Llar de Sabadell (fuente autoras). con el objetivo de re-densificar el barrio. Así como el grado de reducción en las emisiones de $\mathrm{CO} 2$ de las ampliaciones o reformas, en la búsqueda de un crecimiento más sostenible (en la Fig. 13 puede observarse la tabla de consumo según material).

En el resto de ámbitos, se valoran las actuaciones por separado, otorgando un punto a cada una de ellas en relación a: la atención a los materiales, más allá de sus emisiones de $\mathrm{CO} 2$, en aspectos como su optimización, origen reciclado y trazabilidad; energía consumida y demandada por la vivienda; urbanidad, atendiendo a la relación del interior de la vivienda con su jardín y ambos con la calle y parcelas vecinas; habitabilidad, que contempla la iluminación natural y ventilación cruzada; tratamiento de los residuos, especialmente el control aquellos no contemplados a nivel municipal como la captación y reutilización de aguas pluviales o grises; y el fomento de la biodiversidad mediante las infiltraciones al terreno y el aumento de la vegetación (ver tabla en Fig. 10).

En el taller, se escogieron cuatro tipologías de vivienda, las más frecuentes e interesantes, $\mathrm{y}$ en cada una de ellas se ensayaron cuatro soluciones atendiendo a los cuatro nuevos usos anteriormente mencionados, consiguiendo así 


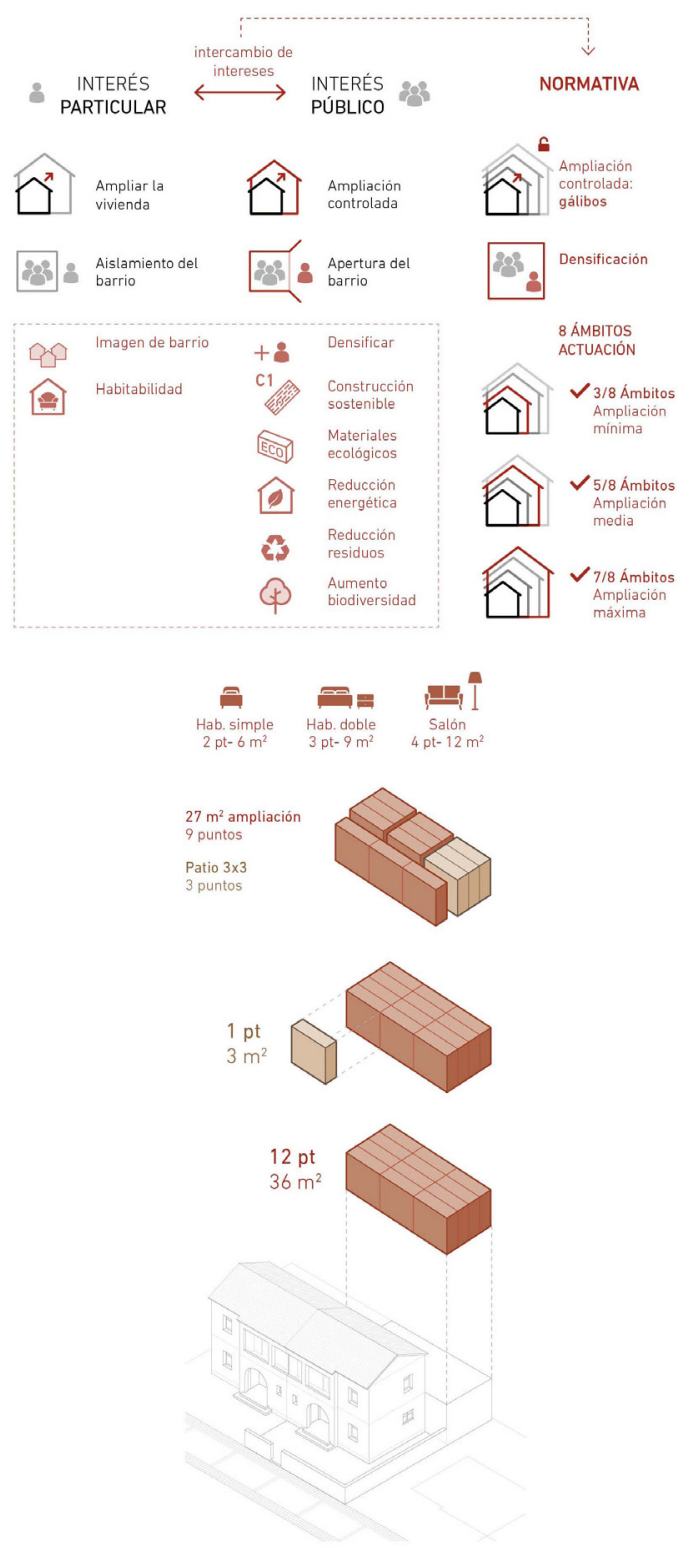

un total de dieciséis casos de estudio de los cuales se exploraron en detalle cuatro, aquellos más complejos y que permitían mostrar un mayor número de soluciones potencialmente extrapolables a las demás viviendas del barrio. Se trata siempre de poner en valor el volumen original de la vivienda, marcando donde se encuentra el límite con la ampliación mediante la materialidad, color o volumetría y permitiendo así una lectura fácil del carácter original del barrio que se había visto alterado (ver algunas intervenciones en perspectiva en la Fig.14).

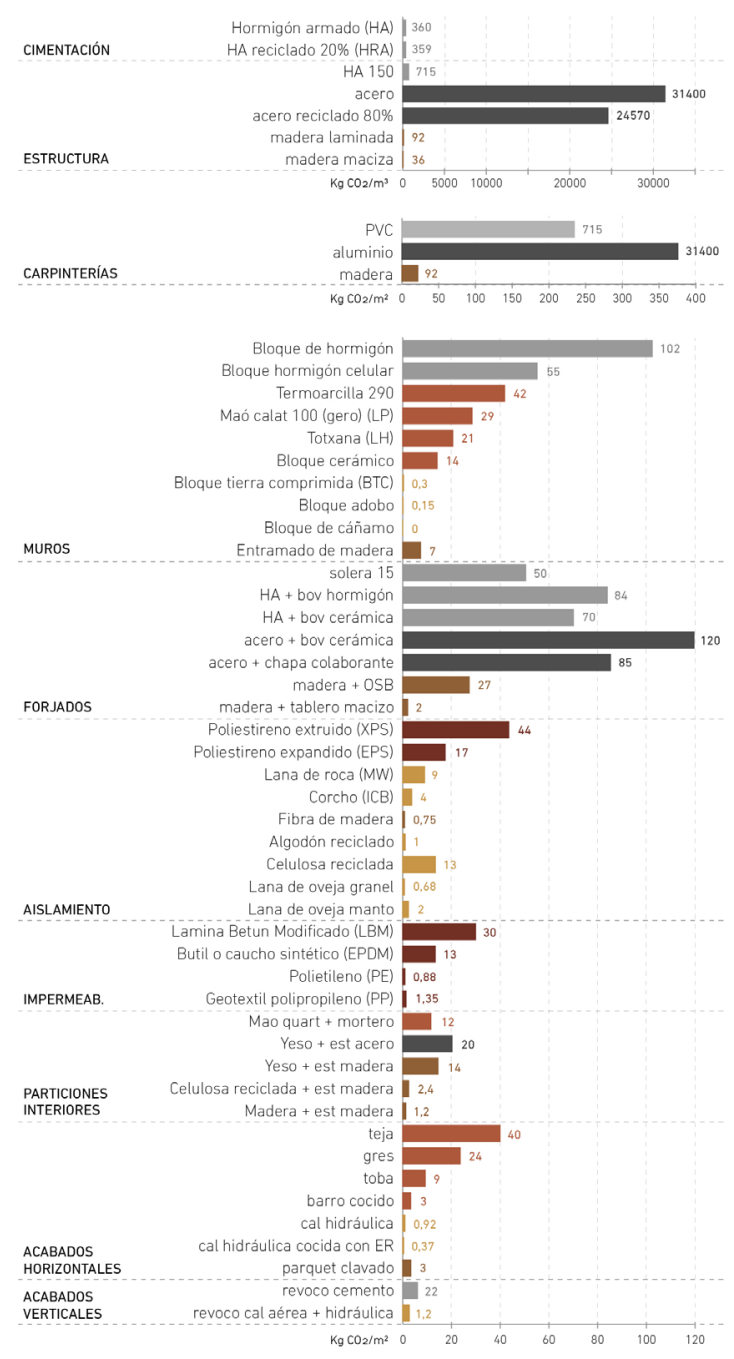

Fig. 12. (izquierda) Esquema conceptual de funcionamiento de la normativa propuesta en el barrio de Nostra Llar de Sabadell (fuente autoras).

Fig. 13. (arriba, derecha) Tabla de consumo de CO2 según material usado en la construcción usado para puntuar las intervenciones en las viviendas y parcelas del conjunto Nostra Llar de Sabadell (fuente autoras).

En conclusión, se genera un catálogo de soluciones útiles para los vecinos de manera que estos puedan contar con una guía que permita que las intervenciones en sus viviendas, aunque progresivas, puedan tener un objetivo final con coherencia formal, a la vez que crezcan de forma sostenible con el medio ambiente y aporten valores positivos a la comunidad.

\section{Conclusión}

El estudio desarrollado en estos tres talleres del Grado y el Máster en Arquitectura de la ETSAV 


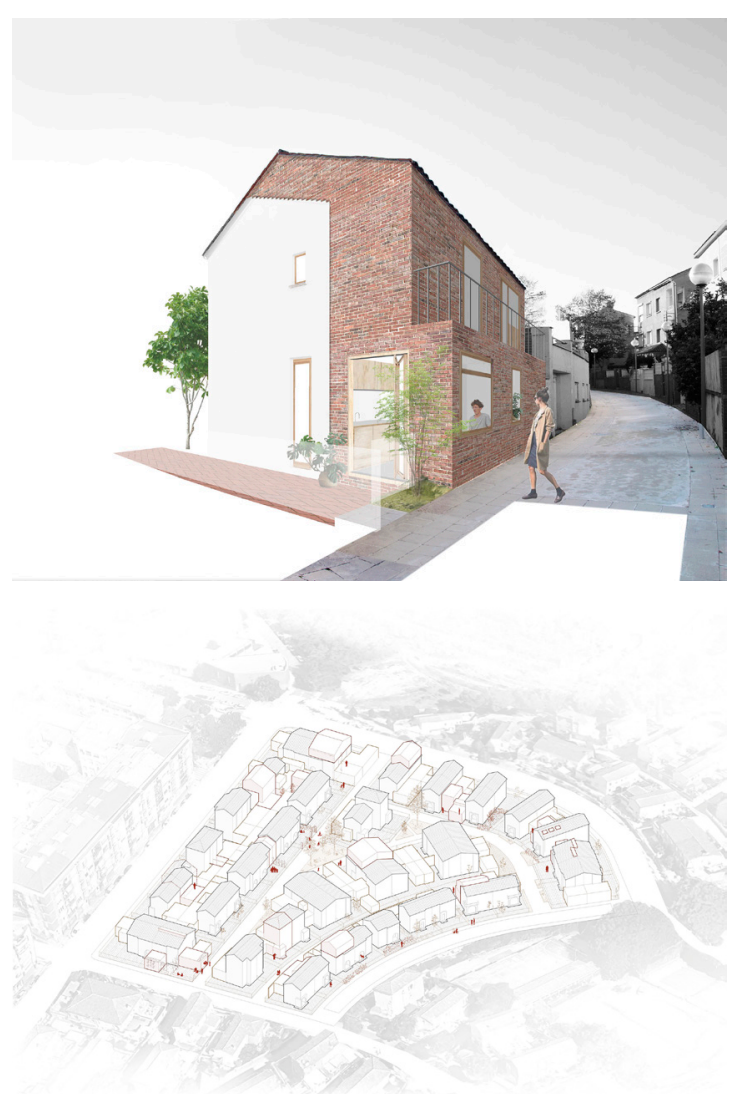

Fig. 14. (arriba) Imagen de algunas de las transformaciones posibles en el barrio de Nostra Llar de Sabadell (fuente autoras).

Fig. 15. (abajo) Perspectiva de conjunto de unos de los sectores de aplicación de la nueva normativa en el barrio de Nostra Llar de Sabadell (fuente autoras).

permiten descifrar una cierta metodología de trabajo para lograr la intensificación de estas áreas de baja densidad vinculadas a los municipios de la Región Metropolitana de Barcelona. Esta metodología consistiría en una primera fase de análisis del área dónde se interviene con el objetivo de, por un lado, conocer el grado de consolidación del parque construido, en referencia al planeamiento vigente, y por el otro, descifrar comportamientos urbanos partiendo de la opinión del vecindario, el análisis de la movilidad, el comercio, el transporte público, detectando calles principales, áreas de centralidad, carencias, oportunidades, para establecer qué estrategia de intensificación debe aplicarse: uniforme, asimétrica, central, lineal.

En este sentido, la ordenanza, bajo la pauta de un Máster Plan, o Plan Especial, que determine las particularidades de planeamiento de cada zona, es una figura ideal para establecer los criterios de bonificación o penalización hacia las intervenciones promovidas por propietarios i/o habitantes con el fin de que, sumando cada una de intervenciones puntuales, se mejore substancialmente los parámetros de sostenibilidad y urbanidad del barrio a cambio del beneficio particular de poder subdividir, vender o alquilar, parte de las viviendas existentes. Estos criterios de bonificación explorados en el caso de Nostra Llar en Sabadell, deberían depender de las particularidades de cada una de estas "urbanizaciones", pero, por lo general, deberían revertir los inconvenientes de la densificación: el aumento de movilidad, gasto energético, residuos o huella ecológica.

En definitiva, la conversión de las "urbanizaciones" en barrios es una buena oportunidad para mejorar la ciudad a partir de la transformación de lo que existe y con la finalidad de encontrar formas de habitabilidad de densidad media, con suficiente masa crítica como para introducir "vida urbana" en estas áreas a menudo patológicamente aisladas, aprovechando el propio metabolismo de crecimiento de sus habitantes y intercambiando ventajas individuales con logros en la sostenibilidad global.

\section{Agradecimientos}

El desarrollo y conclusiones de esta investigación no hubieran sido posibles sin las significativas aportaciones de todo el equipo docente del Taller de Proyectos E1 del Grado en Arquitectura, así como del Máster Universitario en Arquitectura de la ETSAV, especialmente a los profesores Claudi Aguiló, Lorena Maristany y Marta Serra Permanyer que han creído en esta línea de trabajo.

\section{Notas}

1 Ver también el artículo Franquesa 2011 en referencias bibliográficas.

2 Planeamiento aprobado en 2010. http:// territori.gencat.cat/ca/01_departament/ documentacio/territori-i-urbanisme/ ordenacio_territorial/pla_territorial_ambit_ metropolita/

3 Ver Indovina 2007 y Muñoz 2011 en 
referencias bibliográficas. Cabe destacar también el artículo de Francesco Indovina Dalla città diffusa all'arcipelago metropolitano (Indovina 2009).

4 Ver los manuales editados en Estados Unidos Dunhan-Jones 2009 y Tachieva 2010 en referencias bibliográficas.

5 Ver Richard Sennett 2009 en referencias bibliográficas.

6 Sobre vivienda baja densidad ver el articulo Sauquet 2011 en referencias bibliográficas. Sobre urbanismo ecológico partimos de la experiencia docente e investigadora de la Universidad de Harvard recogida en la publicación Urbanismo Ecológico (Mostafavi 2010). Sobre urbanismo ecosistémico tomamos como referencia los parámetros de Ecología Urbana descifrados en la publicación Bettini 1998, en referencias bibliográficas, así como textos de referencia sobre Barcelona de Salvador Rueda.

7 Una publicación tomada de referencia para relacionar materiales de construcción con huella ecológica es $34 \mathrm{Kg}$ de $\mathrm{CO} 2$ (Solanas 2009).

8 El libro La arquitectura de la ciudad, de Aldo Rossi (Rossi 1971) tuvo una gran influencia en los urbanistas encargados de los primeros planes de la democracia española en los años ochenta.

9 La denominada "Escuela de Barcelona" es un movimiento arquitectónico de referencia, que tuvo su relevancia urbanística en los planes para la Barcelona olímpica de 1992, con teóricos de referencia como Manuel de Solá-Morales, Joan Busquets o Oriol Bohigas.

10 Ver Sennet 2014 en referencias bibliográficas.

11 Entender la ciudad como un ser en evolución parte de la idea iniciada por Patrick Geddes a principios de siglo XX en el libro Cities in Evolution (Geddes 2009), así como toda la teoría de la ciudad recogida por Lewis Mumford en sus publicaciones (ver Mumford 2012 en referencias bibliográficas). Igualmente, un plan de la era moderna que comparte estos principios, cuyas conclusiones son muy parecidas a lo que ha significado hoy el sprawl americano, o centroeuropeo, en contraste con otros planes "modernos" como los de Le Corbusier, Sert o Hilberseimer, es el proyecto Bradacre City de Frank Lloyd Wright (ver Wright 1961 en referencias bibliográficas).

12 La "habitación satélite" es un concepto de vivienda no contigua, que tiene asociada un espacio tipo habitación equipada, que puede funcionar autónomamente y ser alquilada. Este concepto parte de la idea explorada por Xavier Monteys en el libro Casa Collage (Monteys 2001) y explicitada posteriormente en la publicación ReHabitar en nueve episodios del grupo HABITAR (HABITAR 2012), liderado por Monteys.

\section{Referencias}

Alexander, Ch. 1969. 3 aspectos de matemática y diseño. Barcelona: Tusquets editor, Cuadernos Ínfimos.

Aravena, A., Iacobelli, A. 2012. Elemental: manual de vivienda incremental y diseño participativo. Ostfildern: Hatje Cantz, coop.

Bettini, V. (ed.). 1998. Elementos de Ecología Urbana. Madrid: Editorial Trotta.

Chermayeff, S., Alexander, Ch. 1968. Comunidad y privacidad. Hacia una nueva arquitectura humanista. Buenos Aires: Ediciones Nueva Visión.

Dunham-Jones, E., Williamson, J., 2009. Retrofitting Suburbia: Urban Design Solutions for Redesigning Suburbs. Ed: N.J. John Wiley \& Sons, Hoboken, NJ.

Franquesa, Jordi. 2011. "Las comunidades jardín: antiguas intuiciones para nuestras nuevas ciudades". Revista Iberoamericana de Urbanismo 5.

Geddes, P. 2009. Ciudades en evolución. Oviedo: KRK Ediciones.

HABITAR, 2012, ReHabitar en nueve episodios. Valencia: Editorial Lampreave.

Indovina, F. (ed.). 2007. La ciudad de baja densidad: lógicas, gestión y contención. Diputació de Barcelona.

Indovina, F. 2009. Dalla città diffusa all'arcipelago metropolitano. Franco Angeli Editori, Milano.

Módenes, J. A., López, J. 2005. "Expansión territorial de la residencia secundaria y ciudad compacta en España: ¿elementos de un mismo sistema?". XXV Conferència 
IUSSP. Tours.

Monteys, X., Fuertes, P. 2001. Casa collage. Un ensayo sobre la arquitectura de la casa. Barcelona: Gustavo Gili.

Mostafavi, M. (ed.). 2010. Ecological Urbanism. Cambridge: Harvard University, Graduate School of Design; Baden: Lars Müller.

Mumford, L. 2012. La ciudad en la historia. Sus orígenes, transformaciones y perspectivas. Logroño: Pepitas de Calabaza.

Muñoz, F. (ed). 2011. Estratègies vers la ciutat de baixa densitat: de la contenció a la gestió. Diputació de Barcelona.

Muxí, Z. (ed.). 2013. Post-Suburbia; Rehabilitación de las urbanitzaciones residenciales monofuncionales de baja densidad. Barcelona: Comanegra.

Rossi, A. 1971. La arquitectura de la ciudad. Barcelona: Gustavo Gili.

Sauquet, R. 2011. "La colectivización de la vivienda en el suburbio de baja densidad. Aproximaciones a propósito del Plan Territorial Metropolitano de Barcelona". Proyecto, Progreso, Arquitectura, n.5, p. 3345.

Sennett, R. 2009, El artesano. Barcelona: Anagrama.

Sennett, R. 2014. L'espai públic. Un sistema obert, un procés inacabat. Barcelona: Arcàdia.

Solanas, T. et al. (ed.) 2009. $34 \mathrm{~kg}$ de CO2. Generalitat de Catalunya, Departament de Medi Ambient i Habitatge.

Tachieva, G. 2010. Sprawl Repair Manual. Washington: Island Press.

Wright, F. L1. 1961. La ciudad viviente. Buenos Aires: Compañía General Fabril Editora. 